\title{
Alda-1 Prevents Pulmonary Epithelial Barrier Dysfunction following Severe Hemorrhagic Shock through Clearance of Reactive Aldehydes
}

\author{
Tianfeng Hua, ${ }^{1,2,3}$ Min Yang, ${ }^{2,3}$ Yangyang Zhou, ${ }^{2,3}$ Limin Chen, ${ }^{2,3}$ \\ Huimei Wu $\mathbb{D}^{1},{ }^{1}$ and Rongyu Liu $\mathbb{1}^{1}$ \\ ${ }^{1}$ Department of Geriatric Respiratory and Critical Care, Anhui Geriatric Institute, The First Affiliated Hospital of \\ Anhui Medical University, Hefei, Anhui, China \\ ${ }^{2}$ The Laboratory of Cardiopulmonary Resuscitation and Critical Care Medicine, The Second Affiliated Hospital of \\ Anhui Medical University, Hefei, China \\ ${ }^{3}$ Intensive Care Unit, The Second Affiliated Hospital of Anhui Medical University, Hefei, China
}

Correspondence should be addressed to Rongyu Liu; rongyuliu@163.com

Received 21 March 2019; Accepted 9 July 2019; Published 4 August 2019

Academic Editor: Christina Pabelick

Copyright (C) 2019 Tianfeng Hua et al. This is an open access article distributed under the Creative Commons Attribution License, which permits unrestricted use, distribution, and reproduction in any medium, provided the original work is properly cited.

\begin{abstract}
Severe hemorrhagic shock and resuscitation (HS/R) can lead to lung injury, resulting in respiratory insufficiency. We investigated whether treatment with Alda-1, an ALDH2 activator, decreased lung injury induced by severe HS/R in a rat model. Male SpragueDawley rats were randomized into three groups, hemorrhagic shock + placebo, hemorrhagic shock + Alda-1, and sham. All animals were heparinized, and then $50 \%$ of the total calculated blood volume was collected over 60 minutes. After 40 minutes of hemorrhagic shock, animals were reinfused with the shed blood over 40 minutes and then observed for an additional 2 hours. Concentrations of 4-HNE, TNF- $\alpha$, IL-6, and ALDH2 activity were detected; lung injury and lung wet-to-dry weight ratios were assessed. Expression of occludin and ZO-1 proteins in lung tissues was also determined. At 2 hours after resuscitation, lung injury was significantly reduced and the wet-to-dry weight ratio was notably decreased in the Alda-1 group compared with placebo $(\mathrm{P}<0.05)$. Alda- 1 treatment also significantly increased the activity of ALDH2 and decreased the levels of toxic 4 -HNE $(\mathrm{P}<0.05)$. In the Alda- 1 group, IL6 and TNF- $\alpha$ were dramatically decreased compared with placebo-treated animals $(\mathrm{P}<0.05)$. Expression of occludin and ZO-1 proteins was significantly decreased in the placebo group compared with the Alda-1 group $(\mathrm{P}<0.05)$. Thus, in a rat model of severe $\mathrm{HS} / \mathrm{R}$, treatment with Alda-1 increased the activity of ALDH2, significantly accelerated the clearance of reactive aldehydes, and concomitantly alleviated lung injury through improvement of pulmonary epithelial barrier integrity resulting in decreased alveolar epithelial tissue permeability, lung edema, and diffuse infiltration of inflammatory cells.
\end{abstract}

\section{Introduction}

Hemorrhage accounts for up to approximately $40 \%$ of trauma-related deaths. Besides its role in early mortality, it is known that severe hemorrhagic shock and resuscitation (HS/R) can result in oxidative stress, which contributes to the development of organ injury, especially in the lungs [1]. Lung injury is the most common cause of respiratory insufficiency after severe hemorrhagic shock and the ensuing resuscitation, which induces infiltration of neutrophils to the lung tissue followed by the release of oxygen radicals and proteases, causing impairment of the surrounding parenchyma cells [2-4] and noncardiogenic pulmonary edema [5]. During severe HS/R, excessive reactive oxygen species (ROS) directly contribute to the initial injury and inflammation [6]. Furthermore, ROS-induced oxidative stress injury can be amplified and propagated by toxic aldehydes produced by ROS-triggered peroxidation of unsaturated lipids [7]. Toxic aldehydes, such as 4-hydroxynonenal (4-HNE), are more stable than ROS, further exacerbating pulmonary injury and enhancing the intense inflammatory response. Therefore, detoxification of reactive aldehydes is an endogenous protective mechanism 
against cell damage resulting from ischemia-reperfusion injury (IRI).

Many studies have shown that aldehyde dehydrogenase 2 (ALDH2) can break down toxic aldehydes into nontoxic substances. Alda-1, the ALDH2 agonist, significantly accelerates the clearance of reactive aldehydes through elevating ALDH2 activity [8-10]. Many studies have demonstrated that Alda1 significantly reduces myocardial, cerebral, and liver injury $[9,11,12]$. Alda-1 also has been demonstrated to alleviate lung ischemia-reperfusion injury [13]. However, the effect of Alda1 on HS/R-induced lung tissue damage is still unknown.

Several recent studies have proposed that injury to the alveolar epithelial barrier increases alveolar permeability, inducing pulmonary edema and subsequent pathological changes. The expression of tight junctions was downregulated in the pathogenesis of acute lung injury and hyperoxiainduced destruction of the pulmonary epithelial barrier [14, 15]. And occludin and zonula occludens 1 [ZO-1] played important functions in maintaining the integrity and barrier function of the tight junctions. Therefore, we hypothesized that increased clearance of reactive toxic aldehydes may decrease injury to the lung tight junctions (occludin and ZO1), maintain the integrity of the pulmonary epithelial barrier, and therefore decrease alveolar epithelial tissue permeability, lung edema, and diffuse infiltration of inflammatory cells. Agents aiming at decreasing toxic aldehydes to protect tight junctions in the alveolar epithelial barrier may be a novel target for prevention and treatment of lung injury induced by severe HS/R. In the present study, we investigated the effect of Alda-1 on lung injury induced by severe HS/R and the potential mechanisms involved.

\section{Materials and Methods}

2.1. Experimental Animals. Twenty healthy male SpragueDawley rats, weighing between 350 and $400 \mathrm{~g}$, were used in this study. All animals were anesthetized by an intraperitoneal injection of pentobarbital $(45 \mathrm{mg} / \mathrm{kg})$ and additional doses of pentobarbital $(10 \mathrm{mg} / \mathrm{kg})$ were required to maintain anesthesia. The trachea of all animals was intubated with the same 14G cannula (Abbocath-T; Abbott Hospital Products Division, North Chicago, IL) which was mounted on a blunt $45^{\circ}$ angled needle. Spontaneous breathing of room air was maintained in all animals and a conventional lead II electrocardiogram was continuously monitored.

Polyethylene catheters (PE-50; Becton Dickinson, Franklin Lakes, NJ) were advanced into the right carotid artery, right femoral artery, and right femoral vein for blood withdrawal, aortic pressure measurement, arterial blood sampling, and blood reinfusion. All catheters were flushed intermittently with saline containing $2.5 \mathrm{IU} / \mathrm{mL}$ of crystalline bovine heparin. A thermocouple microprobe (9030-12-D34; Columbus Instruments, Columbus, $\mathrm{OH}$ ) was inserted into the inferior cava vena from the left femoral vein for measurement of blood temperature, which was maintained at $37^{\circ} \mathrm{C} \pm 0.2^{\circ} \mathrm{C}$ with infrared surface heating lamps.

All experimental animals received humane care in compliance with the Guide for the Care and Use of Laboratory
Animals. This protocol was approved by the Institutional Animal Care and Use Committee of the First Affiliated Hospital of Anhui Medical University.

2.2. Experimental Procedures. Following the above preparation, animals were randomized into three groups: (1) hemorrhagic shock + placebo $(n=8) ;(2)$ hemorrhagic shock + Alda$1(n=8) ;(3)$ sham $(n=4)$. All animals were heparinized with $100 \mathrm{U} / \mathrm{kg}$ bovine heparin. The blood volume of each animal was calculated (estimated blood volume $[\mathrm{EBV}]=6.12 / 100 \mathrm{~g}$ body weight), then an estimated $50 \%$ of the total blood volume was withdrawn over a period of $60 \mathrm{~min}$. Alda-1 (10 mg/kg) was administrated intraperitoneally (i.p.) at the onset of shock. For the placebo group, the same total volume of placebo (50\% polyethylene glycol and 50\% dimethyl sulfoxide) [16] was administered. Animals in the sham group underwent laparotomy but without induction of hemorrhagic shock. Blood from the right carotid artery was let into a sterile $20 \mathrm{ml}$ syringe for storage, using a dual syringe pump (Longer Pump LSP01-1A, Longer Corporation, China). Forty minutes after the completion of bleeding, the animals were reinfused with the shed blood over a period of $40 \mathrm{~min}$. The animals were then monitored for an additional $2 \mathrm{~h}$. Electrocardiography, aortic pressures, and temperature were recorded at $5 \mathrm{~min}$ intervals during the hemorrhage and resuscitation procedures and every $15 \mathrm{~min}$ thereafter. Blood gases and blood samples were collected at baseline, at the end of shock but before reinfusion, and at 1 and $2 \mathrm{~h}$ after the completion of infusion. All animals were euthanized by intravenous injection of pentobarbital $(150 \mathrm{mg} / \mathrm{kg})$ at $2 \mathrm{~h}$ postresuscitation. Routine necropsy was performed for gross documentation of injuries caused by surgical intervention.

2.3. Physiological Measurements. Electrocardiogram and aortic pressure values were continuously recorded on a PCbased data-acquisition system supported by WINDAQ software (DATAQ, Akron, $\mathrm{OH}$ ). Aortic blood $\mathrm{pH}, \mathrm{pCO}_{2}$, $\mathrm{pO}_{2}$, hemoglobin, and lactate concentrations were measured in $0.2 \mathrm{ml}$ blood with a Stat Profile pHOx Plus analyzer (Model RADIOMETER ABL80FLEX; Radiometer Medical ApS, Bronshoj, Denmark). Blood samples were collected from arterial blood and centrifuged at $3000 \times \mathrm{g}$ for $10 \mathrm{mins}$ to obtain serum. Serum and lung samples were stored at $-80^{\circ} \mathrm{C}$ for future analysis. Additionally, lung tissue samples were stored in $4 \%$ paraformaldehyde for histopathology and immunochemistry.

2.4. Lung Wet-to-Dry Weight Ratio. The lung wet-to-dry weight ratio was assessed as previously described [17]. Briefly, after euthanasia, the inferior lobe of the left lung was harvested immediately, then weighed and heated to $65^{\circ} \mathrm{C}$ in a constant temperature oven for $72 \mathrm{hrs}$. The wet-to-dry weight ratio of lungs was calculated at the end of the experiment.

2.5. Lung Histopathologic Analysis. Lung tissue from randomly selected rats within each group was sampled and fixed immediately after the end of each experiment in $4 \%$ paraformaldehyde and then embedded in paraffin. Tissue 
TABLE 1: Baseline characteristics of experimental animals.

\begin{tabular}{lccc}
\hline Measurement & Sham & Hemorrhagic shock + Placebo group & Hemorrhagic shock + Alda-1 group \\
\hline Body weight (g) & $362.50 \pm 6.45$ & $365.00 \pm 9.26$ & $365.00 \pm 10.00$ \\
Heart rate (beats·min-1) & $383.00 \pm 16.46$ & $406.50 \pm 28.46$ & $395.75 \pm 12.88$ \\
MAP (mmHg) & $122.75 \pm 5.68$ & $120.12 \pm 6.87$ & $122.12 \pm 6.49$ \\
Lactate (mmol/L) & $0.90 \pm 0.18$ & $0.84 \pm 0.38$ & $0.80 \pm 0.31$ \\
PH & $7.44 \pm 0.02$ & $7.41 \pm 0.02$ & $7.41 \pm 0.05$ \\
\hline
\end{tabular}

$\mathrm{MAP}$, mean aortic pressure. Values are presented as mean \pm SD.

sections were stained with hematoxylin and eosin (H\&E) and evaluated under light microscopy. The lung histological injury was scored by evaluating the intra-alveolar congestion, intra-alveolar hemorrhage, intra-alveolar, and interstitial infiltration of leukocytes and the thickness of the alveolar wall/hyaline membrane [18]. Twenty high-magnification fields were randomly selected at $\times 400$ magnification within each field. Histopathological evaluation was analyzed by three independent observers who were blinded to the experiment.

2.6. Measurement of 4-HNE Concentration and ALDH2 Activity. 4-HNE concentration and ALDH2 activity were measured using 4-HNE enzyme-linked immunosorbent assay (ELISA) and ALDH2 activity assay kits, respectively (Wuhan CUSABIO Biotech Industry Co. Ltd., China and SigmaAldrich, St. Louis, MO, USA) following the manufacturer's protocols. ALDH2 activity was determined by a coupled enzyme assay in which acetaldehyde is oxidized, generating a colorimetric product proportional to the ALDH2 activity present. The results were read at $450 \mathrm{~nm}$ on a plate reader (TECAN GENios, Austria).

2.7. Cytokine Determination in Lung Tissues. One hundred milligrams of frozen lung tissue were rinsed and homogenized in $1 \mathrm{ml}$ of $1 \times \mathrm{PBS}$ and stored at $-20^{\circ} \mathrm{C}$ overnight. After two freeze-thaw cycles were performed to disrupt the cell membranes, the homogenates were centrifuged at $5000 \times \mathrm{g}$ for $5 \mathrm{~min}$ and the supernatant was then used for cytokine analysis. The cytokines in lung tissue were measured using ELISA kits specific for rat tumor necrosis factor (TNF)- $\alpha$ and interleukin-6 (IL-6) (Wuhan CUSABIO Biotech Industry Co. Ltd., China) following the manufacturer's instructions.

2.8. Occludin and ZO-1 Protein Expression in Lung Tissues. We detected occludin and ZO-1 protein expression by immunohistochemistry. The tissue sections were incubated with antioccludin and anti-ZO-1 antibody (both 1:400, Invitrogen, San Francisco, CA, USA) at $4^{\circ} \mathrm{C}$ overnight and then with a biotinylated secondary antibody. Freshly prepared 3,3'-diaminobenzidine (DAB) was used to visualize the antigen-antibody reaction. Brown granules represented positive cells. Ten high-magnification fields were randomly selected at $\times 400$ magnification within each field. Brown granules were analyzed by three independent observers who were blinded to the experiment.
2.9. Statistical Analysis. Data are presented as mean \pm standard deviation if they were normally distributed. Comparisons between time-based measurements within each group were performed by repeated-measurement analysis of variance. If there was a significant difference in the overall comparison of groups, comparisons among multiple groups were made using a one-way analysis of variance (ANOVA) followed by Scheffe post hoc test. P values of $<0.05$ were considered statistically significant.

\section{Results}

Twenty-three rats were used in this study; however, three of them were excluded for instrumentation or technical failure before randomization. Twenty rats were thus investigated for completion of the experiment. There were no significant differences in baseline characteristics of body weight, body temperature, hemodynamic parameters, blood gas, or arterial blood lactate between the three treatment groups (Table 1).

During the hemorrhage and shock phases, the mean arterial pressure (MAP) was significantly decreased in both HS/R groups when compared with the sham group (all $\mathrm{P}<0.05)$. During the resuscitation phase, MAP was then quickly restored to near-baseline levels. In addition, we found that MAP was significantly better in the Alda-1 group compared with placebo (all $\mathrm{P}<0.05$; Figure 1 ).

3.1. Lung Wet-to-Dry Weight Ratio. The lung wet-to-dry weight ratios were significantly increased at $2 \mathrm{hrs}$ after postresuscitation in both HS/R groups when compared with the sham group (all $\mathrm{P}<0.05)$. However, the wet-to-dry ratio was significantly lower in the Alda-1 group compared with the placebo group $(\mathrm{P}<0.05$; Figure 2$)$.

\subsection{Alda-1 Protected against Lung Tissue Morphological} Injuries. To determine the effects of Alda-1 on lung tissue after severe HS/R, we conducted lung histological analysis by $\mathrm{H} \& \mathrm{E}$ staining (magnification, $\times 400$ ). The lung injury scores were determined by assessing alveolar congestion, exudates, hemorrhage, and infiltration of neutrophils. HS/R induced this lung injury, but administration of Alda-1 ameliorated these indicators of damage, instead demonstrating better aerated alveoli, fewer infiltrating neutrophils and reduced intra-alveolar congestion, exudates, and hemorrhage. The lung injury scores were significantly decreased by $38.7 \%$ in the Alda-1-treated animals compared with the hemorrhagic 


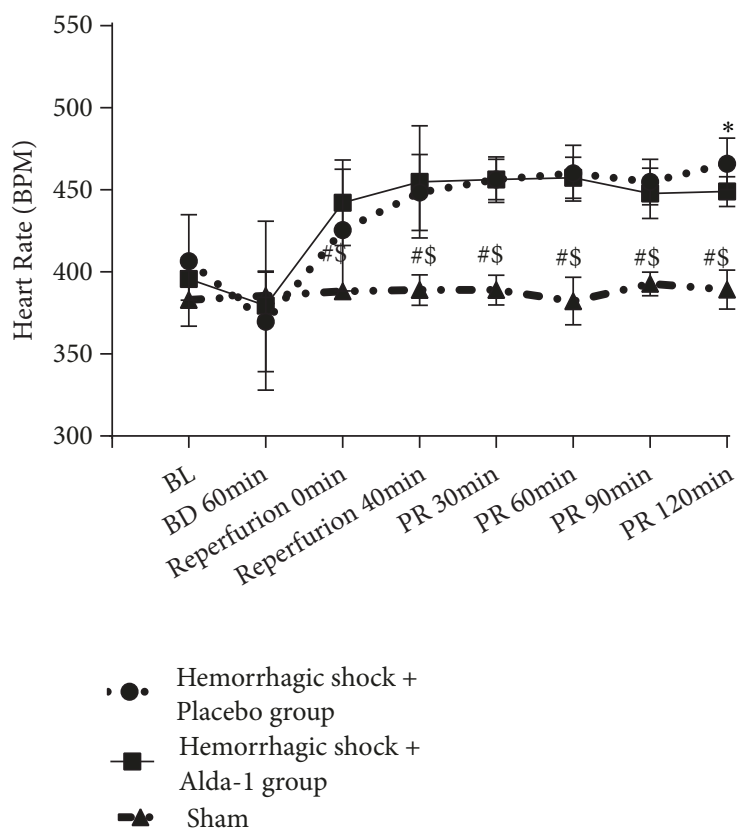

(a)

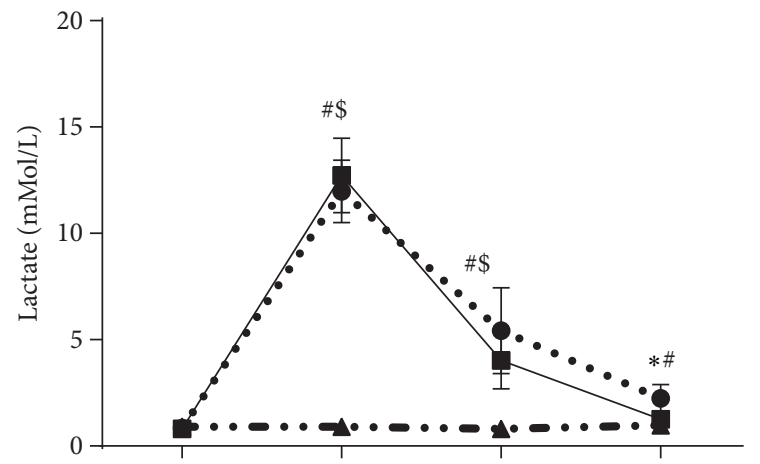

BD<smiles>CC1CCCCC1CCO</smiles>
PR 12000

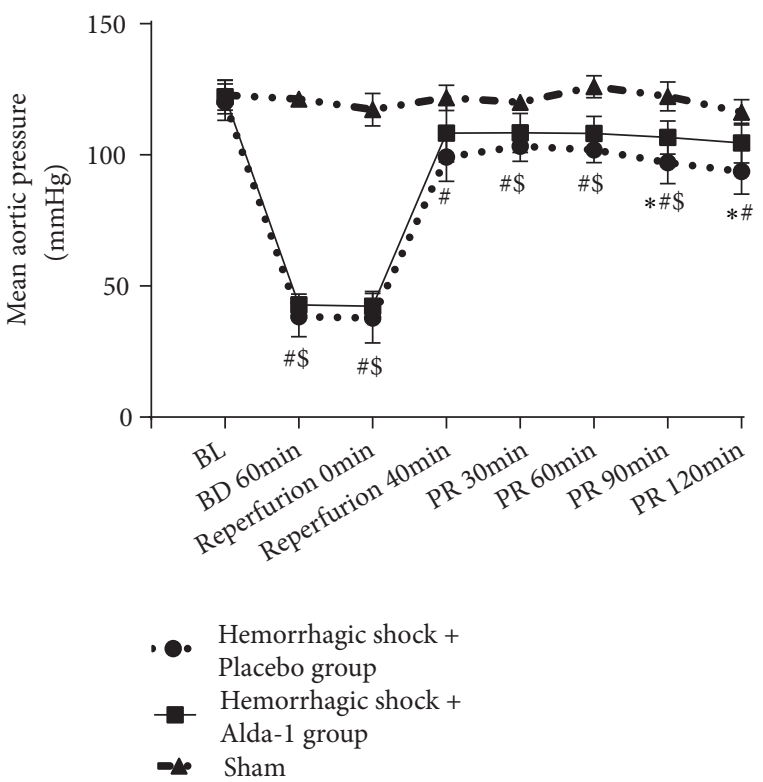

(b)

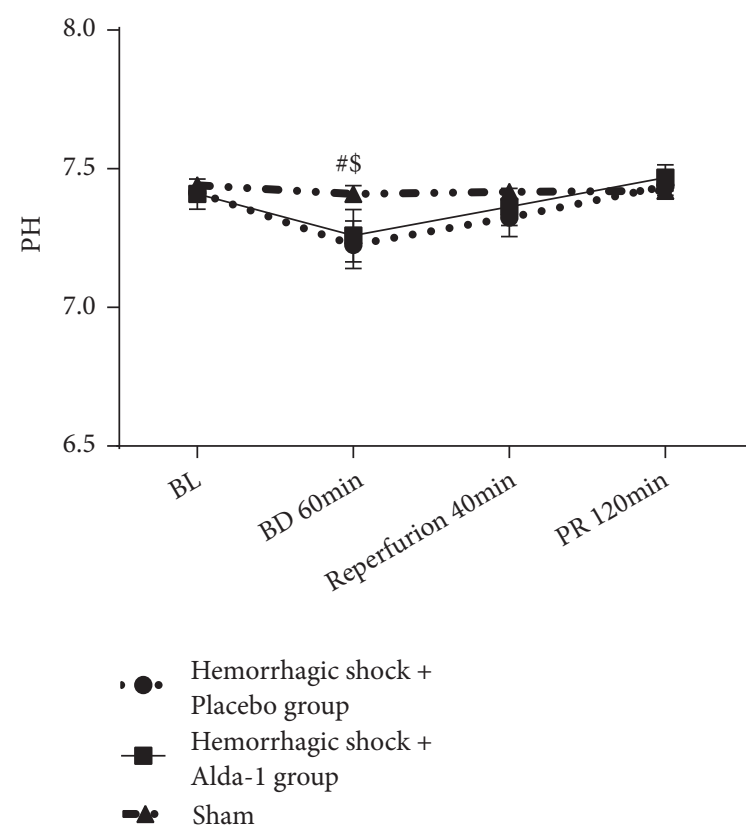

(d)

FIGURE 1: Changes in heart rate (a), mean arterial pressure (b), lactate (c), and $\mathrm{pH}(\mathrm{d})$ during hemorrhagic shock and resuscitation in the three treatment groups, hemorrhagic shock + placebo, hemorrhagic shock + Alda-1 treatment, and sham. BL: baseline; BD: blood withdrawn; $\mathrm{PR}$ : postresuscitation. $* \mathrm{P}<0.05$, hemorrhagic shock + placebo versus hemorrhagic shock + Alda- $1 ; \# \mathrm{P}<0.05$, hemorrhagic shock + placebo versus sham; $\$ \mathrm{P}<0.05$, hemorrhagic shock + Alda-1 versus sham.

shock + placebo group, but increased compared with shamtreated rats $(\mathrm{P}<0.05$; Figure 3$)$.

3.3. Alda-1 Increased ALDH2 Activity and Decreased 4HNE Levels. The data demonstrated that HS/R did not decrease the activity of ALDH2. However, Alda-1 significantly increased the activity of ALDH2. Furthermore, severe HS/R elevated levels of the toxic aldehyde 4-HNE in both shock groups compared with the sham group, but the 4-HNE concentration was dramatically lower in the Alda-1 group compared with placebo-treated rats $(\mathrm{P}<0.05$; Figure 4$)$.

3.4. Alda-1 Decreased IL-6 and TNF- $\alpha$ Levels. The results showed that severe HS/R elevated IL- 6 and TNF- $\alpha$ levels 


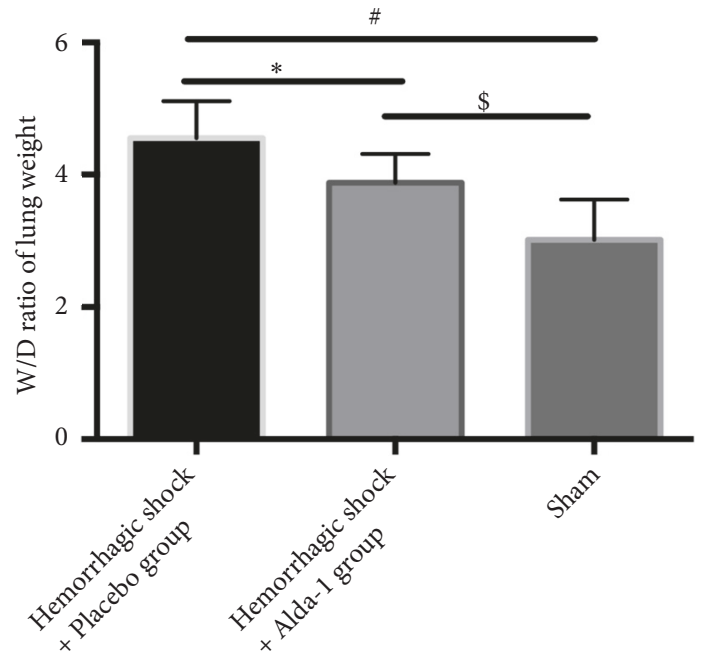

Figure 2: Wet-to-dry (W/D) weight ratio in lung tissue in treated animals. $* \mathrm{P}<0.05$, hemorrhagic shock + placebo versus hemorrhagic shock + Alda-1; $\# \mathrm{P}<0.05$, hemorrhagic shock + placebo versus sham; $\$ \mathrm{P}<0.05$, hemorrhagic shock + Alda-1 versus sham.

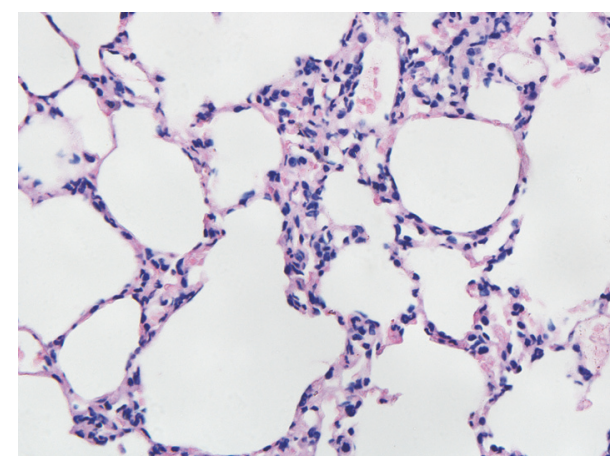

(a)

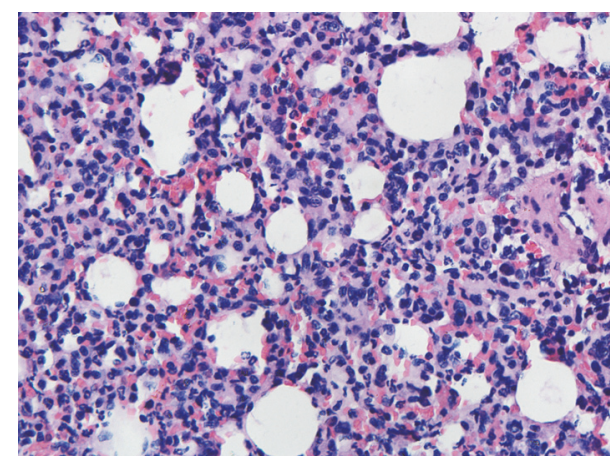

(b)

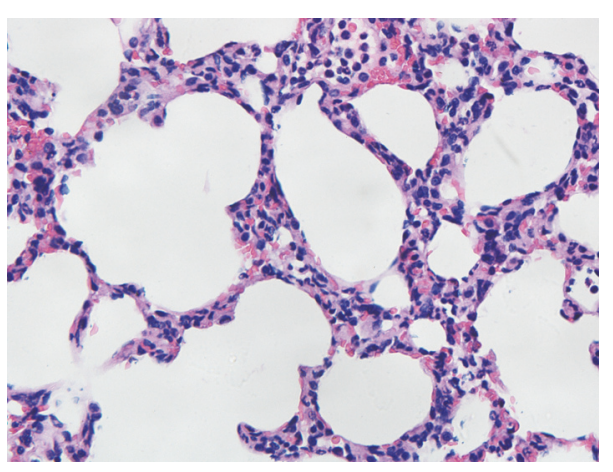

(c)

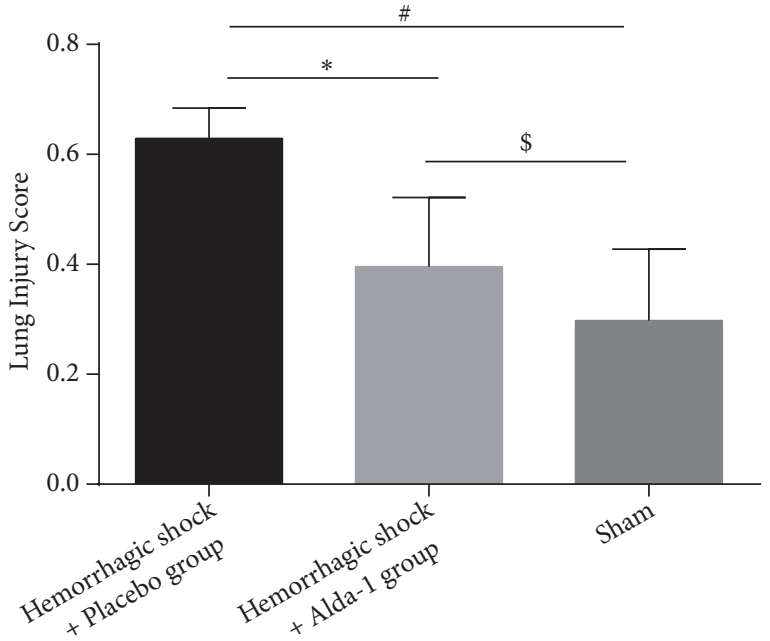

(d)

Figure 3: Lung injury in (a) sham, (b) hemorrhagic shock + placebo, and (c) hemorrhagic shock + Alda-1-treated rats (magnification, $x$ 400). Lung injury was defined as intra-alveolar congestion, intra-alveolar hemorrhage, intra-alveolar and interstitial infiltration of leukocyte infiltration and thickening of the alveolar wall/hyaline membrane. Lung injury scores of sham, hemorrhagic shock + placebo, and hemorrhagic shock + Alda-1 treated rats are presented in $(\mathrm{d}) . * \mathrm{P}<0.05$, hemorrhagic shock + placebo versus hemorrhagic shock + Alda- $1 ; \# \mathrm{P}<0.05$, hemorrhagic shock + placebo versus sham; $\$ \mathrm{P}<0.05$, hemorrhagic shock + Alda-1 versus sham. 


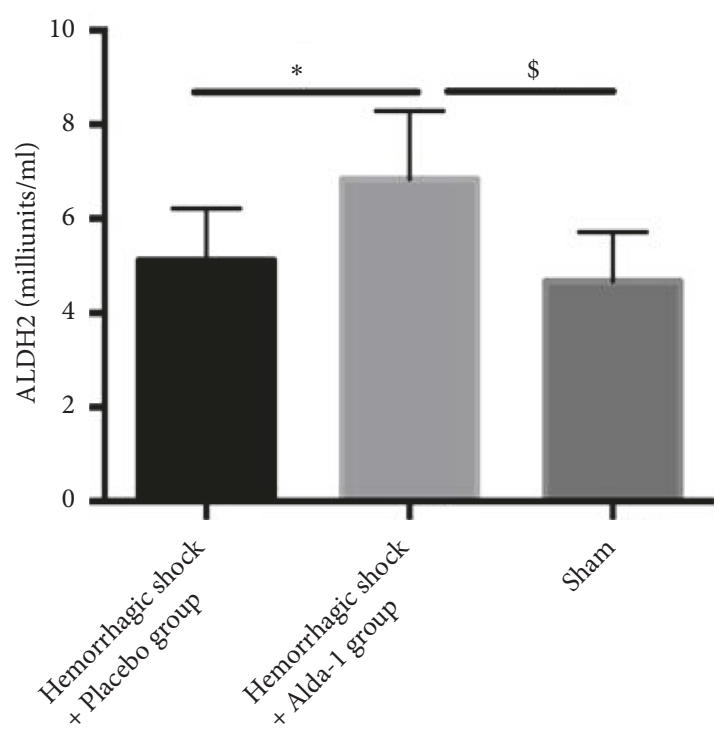

(a)

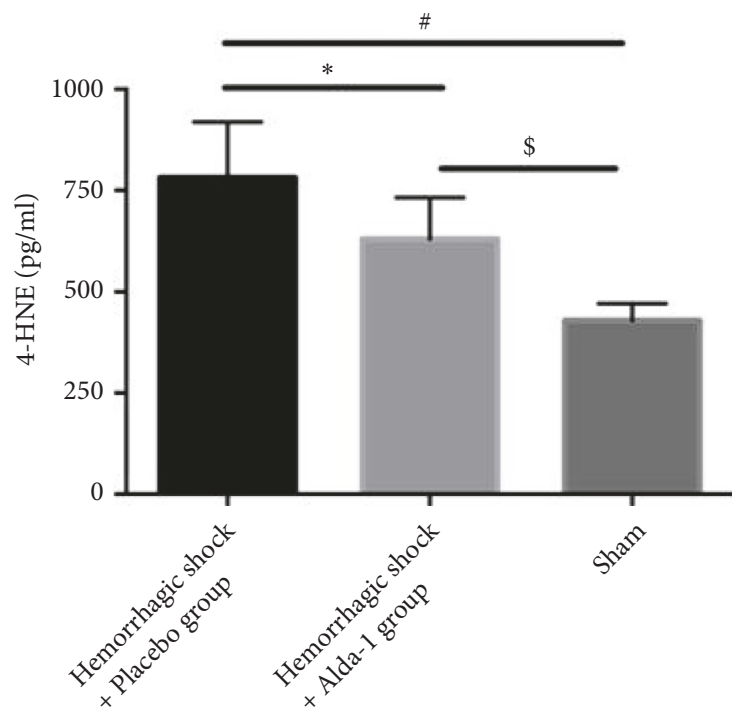

(b)

Figure 4: ALDH2 activity (a) and 4-HNE levels (b) in lung tissues from hemorrhagic shock + placebo, hemorrhagic shock + Alda-1, and sham groups. $* \mathrm{P}<0.05$, hemorrhagic shock + placebo versus hemorrhagic shock + Alda- $1 ; \# \mathrm{P}<0.05$, hemorrhagic shock + placebo versus sham; $\$ \mathrm{P}<0.05$, hemorrhagic shock + Alda-1 versus sham group.

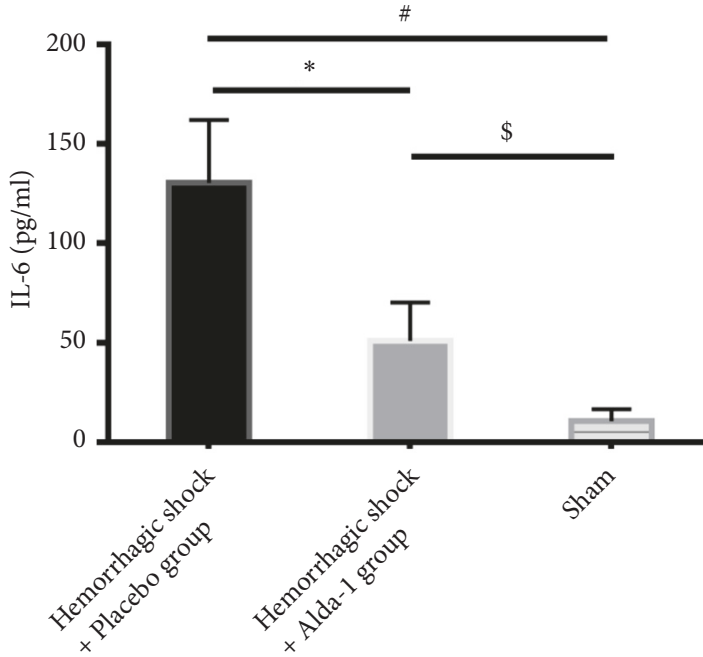

(a)

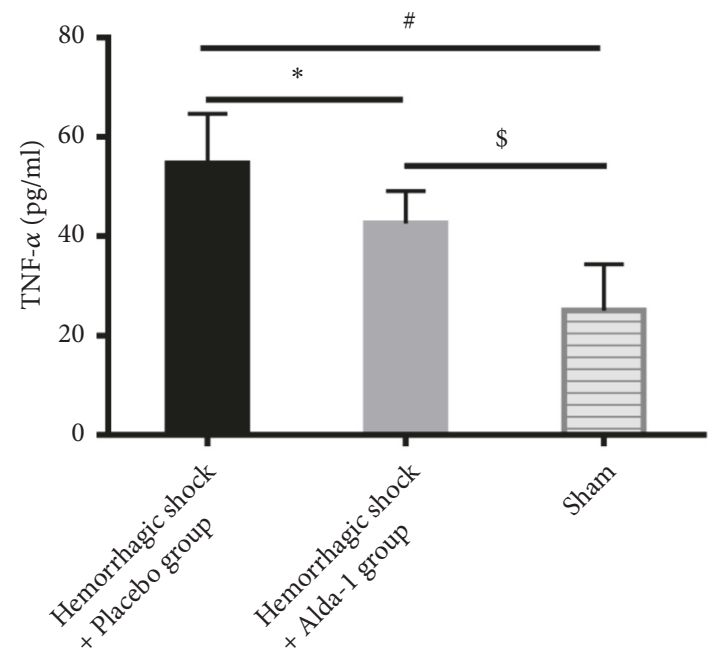

(b)

FiguRE 5: IL-6 (a) and TNF- $\alpha$ (b) levels in lung tissues from hemorrhagic shock + placebo, hemorrhagic shock + Alda-1, and sham groups. $* \mathrm{P}<0.05$, hemorrhagic shock + placebo versus hemorrhagic shock + Alda- $; \# \mathrm{P}<0.05$, hemorrhagic shock + placebo versus sham; $\$ \mathrm{P}<0.05$, hemorrhagic shock + Alda-1 versus sham.

in both shock groups. However, compared with the placebo group, treatment with Alda-1 significantly reduced the IL-6 and TNF- $\alpha$ concentrations ( $<<0.05$; Figure 5$)$.

3.5. Alda-1 Improved Expression of Occludin and ZO-1 Proteins. In order to explain the mechanism of the observed lung-protective effect by Alda-1 in this severe HS/R in vivo model, we investigated the expression of occludin and
Zo-1 proteins, both of which are involved in maintenance of tight junctions in the lung, by immunochemistry. We found that severe HS/R significantly decreased the expression of both occludin and ZO-1 and destroyed the integrity of the pulmonary epithelial barrier. Treatment with Alda-1 significantly increased the expression of these proteins and partially maintained the integrity of pulmonary epithelial barrier $(\mathrm{P}<0.05$; Figure 6$)$. 


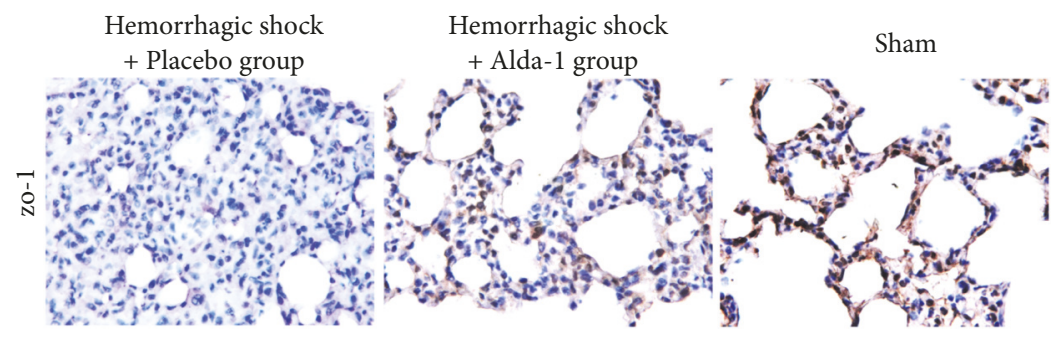

(a)

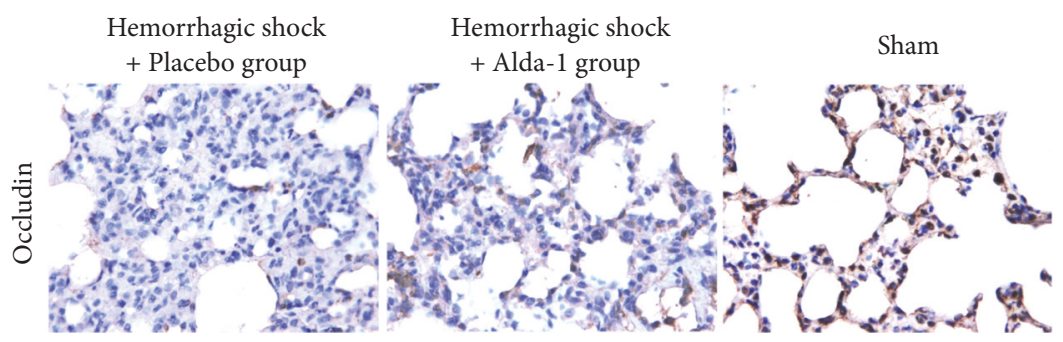

(c)

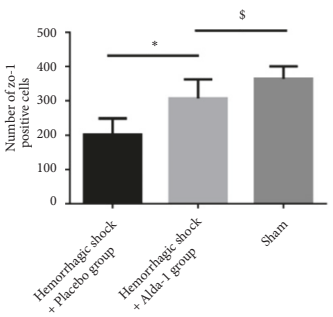

(b)

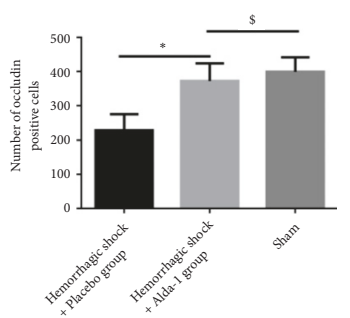

(d)

Figure 6: Expression of ZO-1 (a) and occluding (c) proteins in hemorrhagic shock + placebo, hemorrhagic shock + Alda-1, and sham-treated rats with immunohistochemistry (magnification, $\times 400$ ). ZO-1 and occluding were quantified by brown granules which represent positive cells (b, d). $* \mathrm{P}<0.05$, hemorrhagic shock + placebo versus hemorrhagic shock + Alda-1; $\# \mathrm{P}<0.05$, hemorrhagic shock + placebo versus sham; $\$ \mathrm{P}<0.05$, hemorrhagic shock + Alda-1 versus sham.

\section{Discussion}

In the present study, we have demonstrated that administration of Alda-1 significantly decreases lung injury and alleviates lung edema following severe HS/R. It is likely that the potential mechanism involves Alda-1-mediated induction of ALDH2 activity $[9,10]$ which in turn decreases the accumulation of reactive aldehydes and inflammatory factors, thus significantly attenuating damage to tight junction proteins, ultimately maintaining the integrity of the pulmonary epithelial barrier.

Respiratory insufficiency is the most common and inevitable complication following severe HS/R, during which the imbalance between lung oxygen supply and consumption can lead to excessive oxidative stress. Generation of ROS occurs in most pulmonary cells, including endothelial and alveolar epithelial cells. Furthermore, infiltration of various inflammatory cells, which also produce ROS, further increases the ROS levels in the lung tissue. Ultimately, this not only causes direct damage to local cells and tissues, but also results in the production of toxic aldehydes, which are more stable than ROS and thus further propagate and amplify the oxidative stress injury resulting from ischemia-reperfusion $[11,19]$. Previous studies have proven that 4-HNE levels were positively correlated with IRI [11]. Likewise, we found that 4HNE was significantly increased during severe HS/R, with concomitant increases in histological lung injury score and lung wet-to-dry weight ratio in both groups suffering from severe HS/R when compared with the sham group.

ALDH2, a tetrameric enzyme, detoxificated the toxic aldehydes generated from oxidative stress such as 4 -HNE $[20,21]$. And the levels of reactive acetaldehydes were correlated with outcomes after cardiac I/R injury. Another study further demonstrated that heart protection effect induced by acetaldehydes was through translocating $€ \mathrm{PKC}$ to mitochondria and activating ALDH2 [22]. ALDH2 exists in many organs including the lung $[23,24]$. Here we found that $\mathrm{HS} / \mathrm{R}$ dramatically induced 4 -HNE production; however, the activity of ALDH2 was not significantly decreased within lung tissues during severe H/R. The most likely explanation for the resulting lung damage would be that production of reactive toxic aldehydes such as 4-HNE far surpasses the clearance capacity of ALDH2 during HS/R. Accelerating the removal of aldehydes has been proven to protect the organ from IRI in many animal models. If this also occurs in the lung suffering from HS/R, then it could explain the lung-protective effects of Alda-1 observed in our model. In fact, Alda-1 treatment significantly enhanced the activity of ALDH2, successfully accelerating the clearance of toxic aldehydes, consistent with the results of the wet-to-dry weight ratio, abnormal secretion of inflammatory cytokines and neutrophil infiltration observed in lung tissue $[25,26]$, which supported our hypothesis. To the best of our knowledge, this is the first study to show that activating ALDH2 by administration of Alda-1 could successfully attenuate pulmonary injury induced by severe HS/R.

Additionally, we further explored the potential mechanism of the observed protective effect of Alda-1. The pathogenesis of pulmonary injury that occurs in severe $\mathrm{HS} / \mathrm{R}$ is characterized by increased pulmonary permeability, noncardiogenic pulmonary edema, diffuse infiltration of various inflammatory cells, and pulmonary atelectasis [27]. Previous studies have demonstrated that damage to the alveolar epithelial barrier would lead to such pathogenesis $[14,28,29]$. The alveolar epithelial barrier is composed of a monolayer of alveolar epithelial cells and tight junctions 
between the alveoli and fluid-filled tissue to maintain normal gas exchange. The paracellular tight junctions are responsible for intercellular sealing and regulation of epithelial permeability [15]. Tight junctions include cytoplasmic and transmembrane proteins, together with alveolar epithelial cells to form the alveolar epithelial barrier. ZO-1 and occludin are recognized as two characteristic proteins within tight junctions. Occludin is a transmembrane protein important for stability and barrier function in the tight junction [30, 31]. ZO-1 is an intracellular protein that connects occludin to cytoskeletal proteins and affects paracellular permeability [32]. Disruption of the alveolar epithelial barrier integrity and subsequent increased alveolar epithelial permeability results in alveolar flooding, pulmonary edema, and pathological alterations [33]. Previous studies have demonstrated that 4HNE triggers apoptosis of human pulmonary alveolar cells in vitro and increases pulmonary alveolar capillary barrier permeability [13]. Acrolein has been proven to increase bronchial epithelial barrier permeability [34] and to decrease expression of the tight junction protein Claudin 5 in an endothelial cell line [35]. However, permeability of the alveolar epithelial barrier is lower than that of the vascular endothelial barrier and plays a decisive role in the development of lung edema [15]. Thus, it is essential to investigate the effect of Alda-1 on alveolar epithelial barrier function. In fact, we observed that the expression of occludin and ZO-1 proteins was significantly decreased during severe HS/R and administration of Alda-1 successfully increased expression of these proteins, thus partly alleviating the damage to the alveolar epithelium integrity and protecting alveolar epithelial barrier permeability. This resulted in decreased lung edema and infiltration of neutrophils and ultimately alleviated lung injury.

As demonstrated in our research, severe HS/R significantly elevated the levels of IL- 6 and TNF- $\alpha$ [36]. TNF- $\alpha$ interferes with the transcription and intracellular localization of tight junction proteins [37]. Following the removal of 4-HNE, expression of IL- 6 and TNF- $\alpha$ was significantly decreased. Therefore, we have reason to speculate that 4-HNE also interferes with the expression of tight junction proteins through induction of inflammatory mediators during severe HS/R-induced pulmonary injury.

There are several limitations in our research. Firstly, only healthy male rats without disease were used, which does not reflect the clinical situation of human patients. Secondly, toxicity testing is required before the compound can be progressed for clinical use. Thirdly, the survival rate of animals and larger randomized studies must be investigated.

\section{Conclusions}

In summary, our study demonstrated that treatment with Alda-1 significantly protected the lung against severe HS/Rinduced injury. The potential mechanism could be through activation of ALDH2 to detoxify 4-HNE, thus alleviating damage to tight junctions in the alveolar epithelium and therefore protecting the alveolar epithelial barrier, ultimately leading to reduced lung edema and injury.

\section{Data Availability}

The data used to support the findings of this study are included within the article.

\section{Conflicts of Interest}

All authors declare that they have no conflicts of interest regarding the publication of this paper.

\section{Acknowledgments}

This study was supported by a research grant from the Natural Science Foundation of China [grant numbers 81170030 and 81270082] and the Key Laboratory of Geriatric Molecular Medicine of Anhui Province [grant number 1206c0805028].

\section{References}

[1] D. Safavian, C. H. Leung, A. Kapus et al., "Hemorrhagic shock/resuscitation reduces the M2 phenotype of alveolar macrophages," Shock, vol. 51, no. 2, pp. 213-220, 2019.

[2] D. L. Carden and D. N. Granger, "Pathophysiology of ischaemia-reperfusion injury," The Journal of Pathology, vol. 190, no. 3, pp. 255-266, 2000.

[3] S. D. Ross, C. G. Tribble, J. R. Gaughen, K. S. Shockey, P. E. Parrino, and I. L. Kron, "Reduced neutrophil infiltration protects against lung reperfusion injury after transplantation," The Annals of Thoracic Surgery, vol. 67, no. 5, pp. 1428-1433, 1999.

[4] M. J. Eppinger, G. M. Deeb, S. F. Bolling, and P. A. Ward, "Mediators of ischemia-reperfusion injury of rat lung," The American Journal of Pathology, vol. 150, no. 5, pp. 1773-1784, 1997.

[5] R. Pararajasingam, M. L. Nicholson, P. R. F. Bell, and R. D. Sayers, "Non-cardiogenic pulmonary oedema in vascular surgery," European Journal of Vascular and Endovascular Surgery, vol. 17, no. 2, pp. 93-105, 1999.

[6] W. A. den Hengst, J. F. Gielis, J. Y. Lin, P. E. van Schil, L. J. de Windt, and A. L. Moens, "Lung ischemia-reperfusion injury: a molecular and clinical view on a complex pathophysiological process," American Journal of Physiology-Heart and Circulatory Physiology, vol. 299, no. 5, pp. H1283-H1299, 2010.

[7] B. G. Hill and A. Bhatnagar, "Beyond reactive oxygen species," Circulation Research, vol. 105, no. 11, pp. 1044-1046, 2009.

[8] C. Chen, L. Sun, and D. Mochly-Rosen, "Mitochondrial aldehyde dehydrogenase and cardiac diseases," Cardiovascular Research, vol. 88, no. 1, pp. 51-57, 2010.

[9] C.-H. Chen, G. R. Budas, E. N. Churchill, M.-H. Disatnik, T. D. Hurley, and D. Mochly-Rosen, "Activation of aldehyde dehydrogenase-2 reduces ischemic damage to the heart," Science, vol. 321, no. 5895, pp. 1493-1495, 2008.

[10] S. Perez-Miller, H. Younus, R. Vanam, C. Chen, D. MochlyRosen, and T. D. Hurley, "Alda-1 is an agonist and chemical chaperone for the common human aldehyde dehydrogenase 2 variant," Nature Structural \& Molecular Biology, vol. 17, no. 2, pp. 159-164, 2010.

[11] S. Fu, H. Zhang, Z. Yang et al., "Alda-1 reduces cerebral ischemia/reperfusion injury in rat through clearance of reactive aldehydes," Naunyn-Schmiedeberg's Archives of Pharmacology, vol. 387, no. 1, pp. 87-94, 2014. 
[12] T. Zhang, Q. Zhao, F. Ye, C. Huang, W. Chen, and W. Huang, "Alda-1, an ALDH2 activator, protects against hepatic ischemia/reperfusion injury in rats via inhibition of oxidative stress," Free Radical Research, vol. 52, no. 6, pp. 629-638, 2018.

[13] J. Ding, Q. Zhang, Q. Luo et al., "Alda-1 attenuates lung ischemia-reperfusion injury by reducing 4-hydroxy-2-nonenal in alveolar epithelial cells," Critical Care Medicine, vol. 44, no. 7, pp. e544-e552, 2016.

[14] K. You, X. Xu, J. Fu et al., "Hyperoxia disrupts pulmonary epithelial barrier in newborn rats via the deterioration of occludin and ZO-1," Respiratory Research, vol. 13, no. 1, p. 36, 2012.

[15] J. Yang, Y. Wang, H. Liu, J. Bi, and Y. Lu, "C2-ceramide influences alveolar epithelial barrier function by downregulating Zo1, occludin and claudin-4 expression," Toxicology Mechanisms and Methods, vol. 27, no. 4, pp. 293-297, 2017.

[16] K. M. S. Gomes, L. R. Bechara, V. M. Lima et al., "Aldehydic load and aldehyde dehydrogenase 2 profile during the progression of post-myocardial infarction cardiomyopathy: benefits of Alda-1," International Journal of Cardiology, vol. 179, pp. 129-138, 2015.

[17] A. D. Rafie, P. A. Rath, M. W. Michell et al., "Hypotensive resuscitation of multiple hemorrhages using crystalloid and colloids," Shock, vol. 22, no. 3, pp. 262-269, 2004.

[18] G. Matute-Bello, G. Downey, B. B. Moore et al., "An official american thoracic society workshop report: features and measurements of experimental acute lung injury in animals," American Journal of Respiratory Cell and Molecular Biology, vol. 44, no. 5, pp. 725-738, 2011.

[19] R. A. Vaishnav, I. N. Singh, D. M. Miller, and E. D. Hall, "Lipid peroxidation-derived reactive aldehydes directly and differentially impair spinal cord and brain mitochondrial function," Journal of Neurotrauma, vol. 27, no. 7, pp. 1311-1320, 2010.

[20] S. Josan, T. Xu, Y.-F. Yen et al., "In vivo measurement of aldehyde dehydrogenase-2 activity in rat liver ethanol model using dynamic MRSI of hyperpolarized [1-13C]pyruvate," NMR in Biomedicine, vol. 26, no. 6, pp. 607-612, 2013.

[21] D. R. Petersen and J. A. Doorn, "Reactions of 4-hydroxynonenal with proteins and cellular targets," Free Radical Biology \& Medicine, vol. 37, no. 7, pp. 937-945, 2004.

[22] C. B. Ueta, J. C. Campos, R. P. Albuquerque et al., "Cardioprotection induced by a brief exposure to acetaldehyde: role of aldehyde dehydrogenase 2," Cardiovascular Research, vol. 114, no. 7, pp. 1006-1015, 2018.

[23] M. J. Stewart, K. Malek, and D. W. Crabb, "Distribution of messenger RNAs for aldehyde dehydrogenase 1, aldehyde dehydrogenase 2, and aldehyde dehydrogenase 5 in human tissues," Journal of Investigative Medicine, vol. 44, no. 2, pp. 4246, 1996.

[24] M. Yoon, M. C. Madden, and H. A. Barton, "Developmental expression of aldehyde dehydrogenase in rat: a comparison of liver and lung development," Toxicological Sciences, vol. 89, no. 2, pp. 386-398, 2006.

[25] J. J. Coalson, "Pathology of new bronchopulmonary dysplasia," Seminars in Fetal and Neonatal Medicine, vol. 8, no. 1, pp. 73-81, 2003.

[26] B. B. Warner, L. A. Stuart, R. A. Papes, and Wispe. J. R., "Functional and pathological effects of prolonged hyperoxia in neonatal mice," American Journal of Physiology, pp. 275-110, 1998.

[27] R. S. Ferrari and C. F. Andrade, "Oxidative stress and lung ischemia-reperfusion injury," Oxidative Medicine and Cellular Longevity, vol. 2015, Article ID 590987, 14 pages, 2015.
[28] E. Crandall and M. Matthay, "Alveolar epithelial transport," American Journal of Respiratory and Critical Care Medicine, vol. 163, no. 4, pp. 1021-1029, 2001.

[29] L. B. Ware and M. A. Matthay, "Alveolar fluid clearance is impaired in the majority of patients with acute lung injury and the acute respiratory distress syndrome," American Journal of Respiratory and Critical Care Medicine, vol. 163, no. 6, pp. 13761383, 2001.

[30] K. M. McCarthy, I. B. Skare, M. C. Stankewich et al., "Occludin is a functional component of the tight junction," Journal of Cell Science, vol. 109, no. 9, pp. 2287-2298, 1996.

[31] Y. Chen, C. Merzdorf, D. L. Paul, and D. A. Goodenough, " $\mathrm{COOH}$ terminus of occludin is required for tight junction barrier function in early Xenopus embryos," The Journal of Cell Biology, vol. 138, no. 4, pp. 891-899, 1997.

[32] B. M. Denker and S. K. Nigam, "Molecular structure and assembly of the tight junction," American Journal of Physiology, vol. 274, pp. 1-9, 1998.

[33] S. Yanagi, H. Tsubouchi, A. Miura, N. Matsumoto, and M. Nakazato, "Breakdown of epithelial barrier integrity and overdrive activation of alveolar epithelial cells in the pathogenesis of acute respiratory distress syndrome and lung fibrosis," BioMed Research International, vol. 2015, Article ID 573210, 12 pages, 2015.

[34] R. C. Grafström, J. M. Dypbukt, J. C. Willey et al., "Pathobiological effects of acrolein in cultured human bronchial epithelial cells," Cancer Research, vol. 48, no. 7, pp. 1717-1721, 1988.

[35] Q. Lu, M. Mundy, E. Chambers et al., "Alda-1 protects against acrolein-induced acute lung injury and endothelial barrier dysfunction," American Journal of Respiratory Cell and Molecular Biology, vol. 57, no. 6, pp. 662-673, 2017.

[36] J. Huang, D. Xu, Q. Guo et al., "Remote ischemic postconditioning improves myocardial dysfunction via the risk and safe pathways in a rat model of severe hemorrhagic shock," Shock, vol. 49, no. 4, pp. 460-465, 2018.

[37] C. B. Coyne, M. K. Vanhook, T. M. Gambling et al., "Regulation of airway tight junctions by proinflammatory cytokines," Molecular Biology of the Cell (MBoC), vol. 13, no. 9, pp. 3218$3234,2002$. 


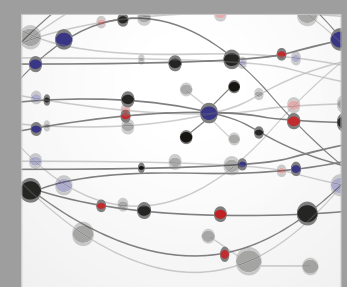

The Scientific World Journal
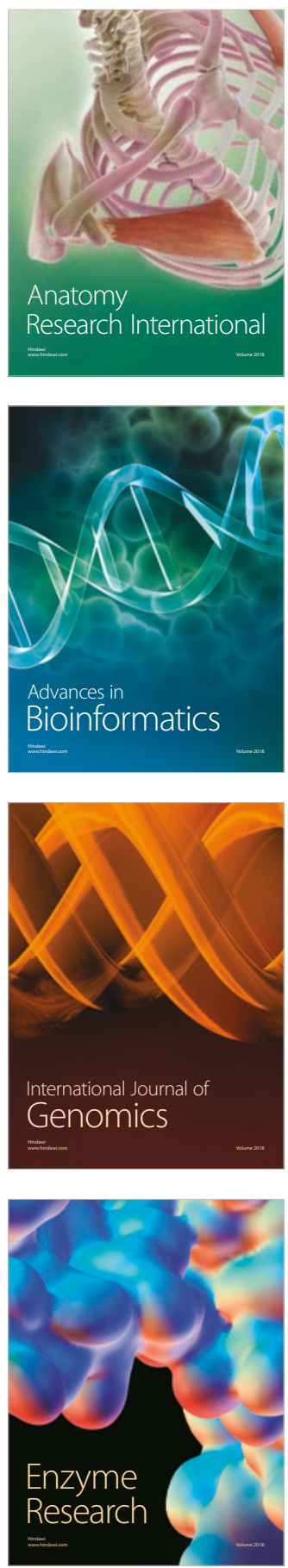
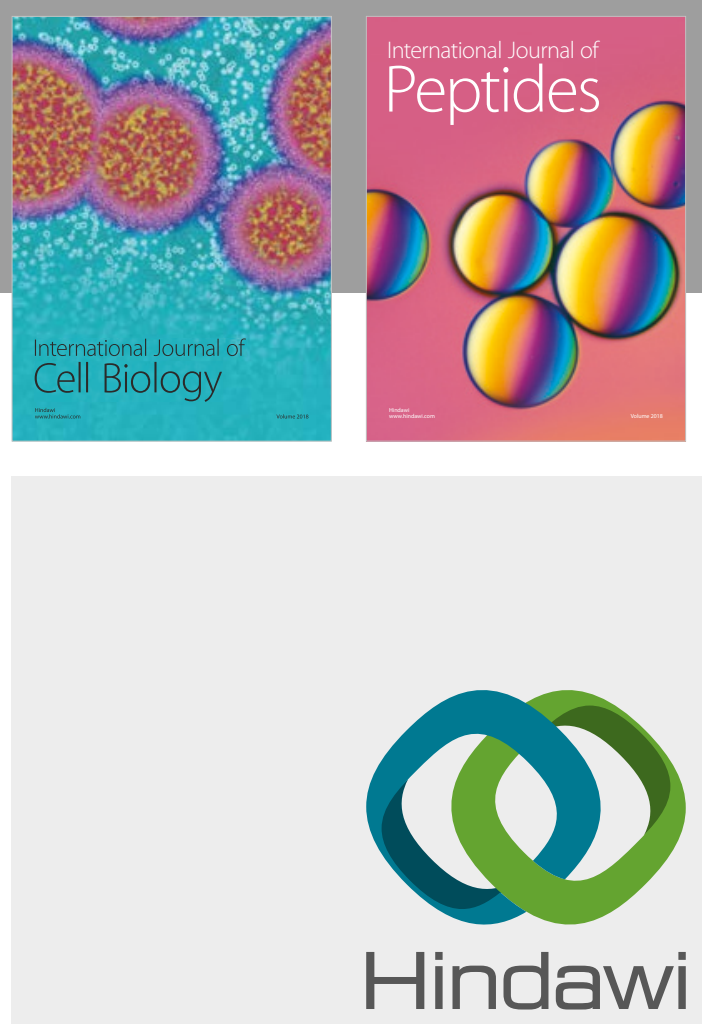

Submit your manuscripts at

www.hindawi.com
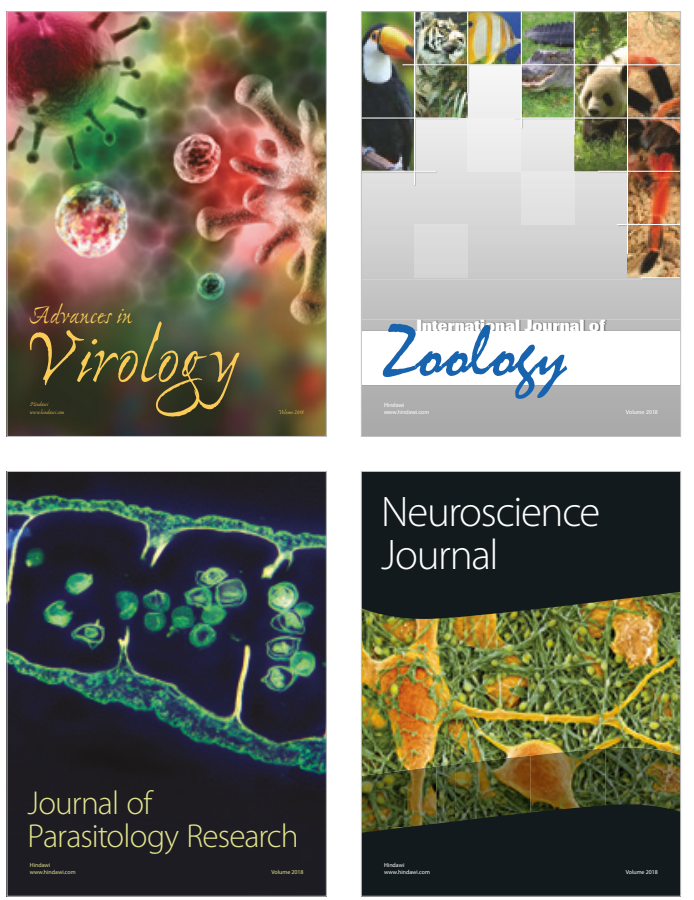
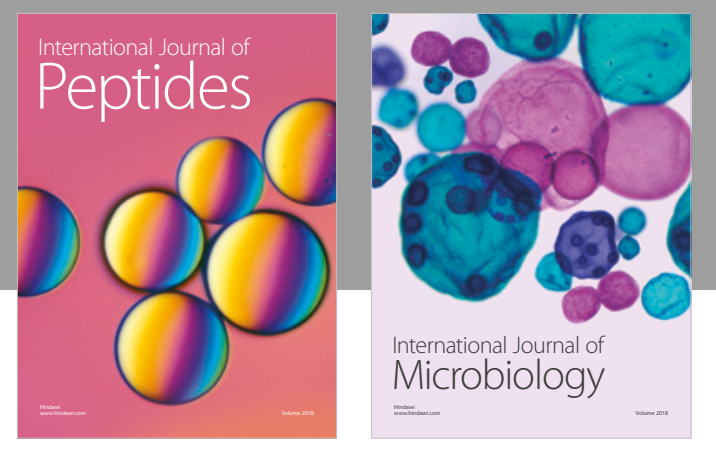

nternational Journal of Microbiology
Journal of
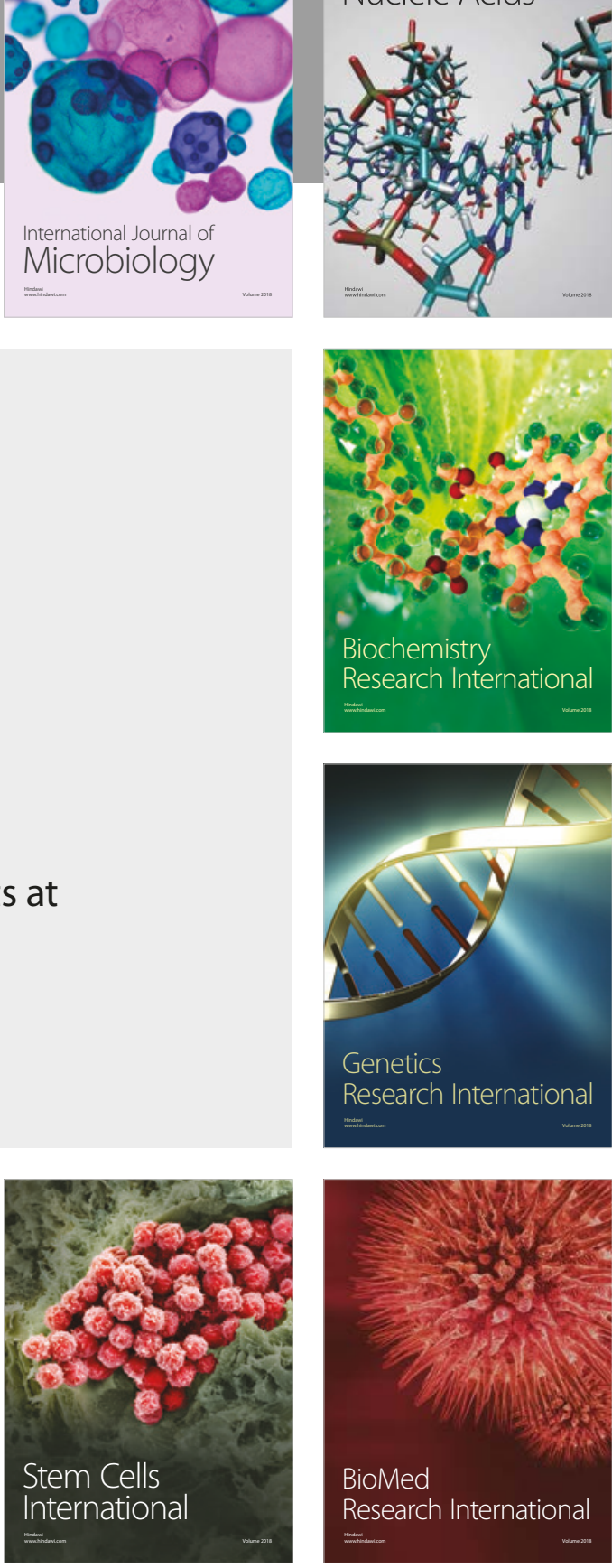
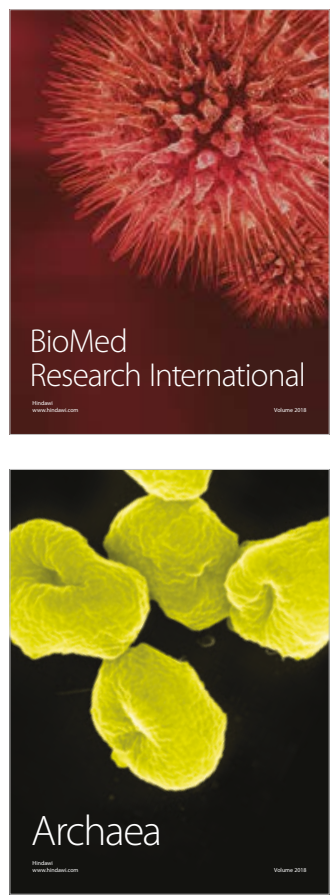